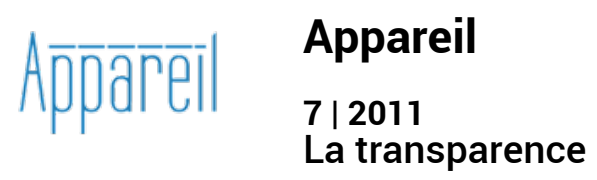

\title{
L'opacité ontologique de la transparence chez Heidegger
}

Hervé Bonnet

\section{OpenEdition}

Journals

Édition électronique

URL : http://journals.openedition.org/appareil/1188

DOI : 10.4000/appareil. 1188

ISSN : 2101-0714

Éditeur

MSH Paris Nord

Référence électronique

Hervé Bonnet, «L'opacité ontologique de la transparence chez Heidegger », Appareil [En ligne], 7 | 2011, mis en ligne le 12 avril 2011, consulté le 30 juillet 2020. URL : http://journals.openedition.org/appareil/ 1188 ; DOI : https://doi.org/10.4000/appareil.1188

Ce document a été généré automatiquement le 30 juillet 2020.

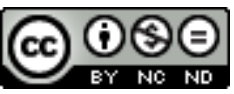

Appareil est mis à disposition selon les termes de la Licence Creative Commons Attribution - Pas d'Utilisation Commerciale - Pas de Modification 4.0 International. 


\title{
L'opacité ontologique de la transparence chez Heidegger
}

\author{
Hervé Bonnet
}

\section{NOTE DE L'AUTEUR}

Utilisant la traduction de Emmanuel Martineau, signalons dès l'abord que le mode d'être de « l'être-à-portée-de-la-main » traduit la Zuhandenheit et celui de « l'être-sousla-main » la Vorhandenheit.

1 S'il fallait établir une topographie terminologique explicitant les concepts fondamentaux de la langue heideggerienne telle qu'elle se déploie à l'occasion de Sein und Zeit (auquel nous limiterons notre investigation) la transparence ferait sans doute partie de ceux qui (ne) seraient évoqués (qu') à l'occasion de l'analyse d'un concept ou d'un thème jugés «majeurs » plutôt que de recevoir un traitement singulier et circonstancié. La raison en est que, d'une part, ce terme, spécifiant la vue propre du Dasein en tant qu'il se comprend authentiquement, ne fait pas l'objet d'une réitération soutenue qui lui accorderait le statut de thème récurrent dans l'œuvre heideggerienne à l'instar de l'être-pour-la-mort, de l'être-à-portée-de-la-main ou du Souci, et d'autre part, ne semble pas communiquer étroitement avec le corpus des concepts heideggerien ni avoir de résonance remarquable au sein de Sein und Zeit.

2 Il en est vraisemblablement ainsi. Mais ce qui semble vrai, pour autant qu'il semble, ne l'est pas nécessairement ni toujours. Or, s'il faut attendre le paragraphe 31 traitant du «Dasein comme comprendre » pour que la signification de la transparence change de destination et passe de sa détermination usuelle à son acception strictement heideggerienne qualifiant désormais une modalité existentiale authentique, il n'en reste pas moins que la transparence, entendue semble-t-il, cette fois, selon son acception courante, fait son apparition dès le début du chef d'œuvre heideggerien lors de la question de « la structure formelle de la question de l'être » au paragraphe 2. 
Heidegger attire notre attention sur le fait que la question de l'être et plus précisément la question du sens de l'être n'est pas une question comme une autre dans la mesure où s'interroger à son sujet nécessite de se questionner préalablement sur le questionnement même, sur la forme même, partant le sens même, de la question. En effet, la question, tout questionner en général présuppose une entente implicite de ce qu'être signifie. Mais cette entente implicite est obscure, vague et laisse l'être dans l'indétermination. C'est pourquoi il est requis une "transparence appropriée" (angemessenen Durchsichtigkeit) afin que cette question principielle par excellence, engageant l'essence même de la question, reçoive une détermination positive. Mais que faut-il entendre par "transparence appropriée " ? Cela implique que la question de l'être puisse faire l'objet d'une transparence inadéquate, impropre. C'est en effet une telle pseudo-transparence qui a, comme l'indiquent les premières phrases de Sein und Zeit, plongé l'être dans l'oubli en le faisant passer pour quelque chose d'évident (Selbsverständliche) et par conséquent d'inquestionnable. D'où la recommandation de Heidegger nous enjoignant de nous tenir à distance des "théories ou des opinions traditionnelles » qui, de façon "inapparente » (verborgen), structurent le discours dominant sur l'être et, au lieu d'engager une compréhension authentique de l'être, " racontent des histoires ». On se raconte des histoires à propos de l'être dès lors que l'on rabat l'être sur l'étant, c'est-à-dire que l'on réduit la compréhension de l'être à celle de l'étant quel qu'il soit. Et pourtant le questionnement lui-même, en tant qu'il est une modalité de l'étant qui questionne, implique que l'on se meuve toujours déjà dans la dimension ontique. Mais cet horizon ontique qui circonscrit tout questionnement ne borne pas pour autant notre compréhension de l'être à celle de l'étant, bref ne nous interdit pas d'interroger l'être en tant qu'être de l'étant. C'est la raison pour laquelle une «transparence appropriée » s'avère nécessaire pour que l'être soit proprement appréhendé au fil d'une question fondamentale et ontologique et non simplement éludé sous couleur d'« évidence».

4 Mais qu'en est il positivement de cette «transparence appropriée »? Puisque nous évoluons d'emblée et pour toujours dans l'élément ontique, rendre sa transparence propre à la question de l'être exige que l'étant pour lequel l'être fait question, l'étant questionnant, soit en son être élucidé. Par conséquent l'étant questionnant est aussi questionné mais non pas en tant qu'étant parmi les étant mais en tant qu'il a rapport à l'être et premièrement à son être. Cet étant nous le sommes nous-mêmes et Heidegger le désigne du nom de Dasein :

cet étant que nous sommes toujours nous-mêmes et qui a entre autres la possibilité essentielle du questionner, nous le saisissons terminologiquement comme Dasein. La position expresse et transparente de la question du sens de l'être exige une explication préalable adéquate d'un étant (le Dasein) au point de vue de son être ${ }^{1}$.

Que la transparence ontologique, proprement réalisée, requiert une transparence ontico-ontologique cela indique une certaine "communauté » de la "propreté » de l'être et de l'être du Dasein au sens où, étrangement, nous assistons à un partage du propre c'est-à-dire un partage de ce qui ne peut être partagé. Plus tard, Heidegger parlera de « copropriété » pour expliciter le rapport singulier et privilégié entre l'être et le Dasein.

6 Si l'accès au sens de l'être est conditionné par une explicitation de l'être du Dasein on comprend alors que la «transparence appropriée » dont il est question fait signe vers une clarification de la modalité ontologico-existentiale du Dasein. Dès lors, on voit se dessiner le lien essentiel qui relie cette «transparence appropriée» qu'exige la 
question du sens de l'être à la transparence du Dasein telle qu'elle est formulée au § 31 . Mieux encore, celle-ci donne accès à celle-là. Cependant, ce n'est qu'au fil d'une interrogation portant sur la question du sens de l'être que la transparence existentiale apparaît comme réquisit nécessaire à la transparence ontologique. Le cercle qui apparaît ici et qui semble vicier le raisonnement l'accrédite en réalité et témoigne en faveur de sa pertinence. En effet, ce n'est pas un hasard si le $\S 2$ et le $\$ 32$ (paragraphe poursuivant l'analyse du «comprendre » engagée au \$ 31) mentionnent ce phénomène bien connu du « cercle logique » et le récusent en indiquant le caractère éminemment existential que ce dernier recouvre en réalité. Si un "cercle vicieux» accompagne la question de l'être ou la question du comprendre du Dasein ce n'est pas qu'il y ait là quelque présupposé illégitime mais cela révèle la structure ontologique circulaire du Dasein. La présence de ce cercle atteste donc un effet positif résultant du questionnement de l'existence eu égard à l'être.

7 Partant, la «transparence » relative à la question de l'être trouve son «appropriement » dans une clarification de l'être du Dasein. Et si, comme l'indique le $\S 6$, la transparence, pour être conquise, implique la destruction de l'histoire de l'ontologie on voit alors se croiser et s'enchevêtrer le thème de la destruction et celui de l'analytique existentiale et se former la trame de la phénoménologie heideggerienne. La transparence ontologique, via la destruction de l'histoire de l'ontologie, ne peut être faite que si le Dasein est, en son être, transparent. Mais quelle est la nature du lien entre l'histoire de l'être et la transparence daseinologique ? Et que veut dire, pour le Dasein, être "transparent »? Heidegger comprend la transparence du Dasein comme la vue propre de ce dernier tel qu'il se comprend en son être, plus précisément, il s'agit d'« une saisie compréhensive de l'ouverture pleine de l'être-aumonde à travers ses moments constitutifs essentiels ${ }^{2}$.» Il s'agit donc de la modalité selon laquelle le Dasein appréhende existentialement et pleinement sa constitution fondamentale.

8 L'expression «à travers " (hindurch), soulignée en italique par l'auteur, indique qu'il faille prendre au sens propre la notion de «traversée» que la définition du " comprendre " comporte et que l'on retrouve dans le préfixe du concept de transparence «Durchsichtigkeit » au sens où est déclaré transparent ce qui « laisse passer » la lumière, ce qui se laisse, par elle, traverser. Il faut donc penser ensemble la transparence, la traversée et la lumière étant donné que la transparence est la traversée de la lumière. Or, la lumière, dans l'économie de la pensée heideggerienne, n'est rien d'autre que la lumen naturale, autrement dit, l'expression de la raison humaine, ou plutôt, selon la terminologie existentiale, le fait que le Dasein est originairement, en son être, éclairci au sens où il est lui-même l'éclaircie. Le Dasein est donc traversé par la propre lumière qu'il est pour lui-même. C'est pourquoi en tant que " comprendre" le Dasein est dans la transparence de son rapport à Soi. Cela, bien entendu, n'a pas valeur d'une claire compréhension de son sens d'être mais indique la singularité ontologique du Dasein en tant qu'il a le privilège d'être toujours déjà l'étant qui est donné à lui-même. Ce qui est donné est donc ce que Heidegger appelle la mienneté du Dasein à savoir le fait que ce dernier est remis à lui-même quant à son être. Une telle donation constitue le fonds sur lequel la possibilité d'acquérir une transparence au niveau du sens de l'être demeure envisageable. Traversé par sa propre lumière, par son autosaisie toujours en cours, signe de l'auto-donation dont il est le 
bénéficiaire, le Dasein est l'étant pour lequel l'être fait sens, en un mot, pour qui l'être est.

Mais si le Dasein est constamment traversé par cette "conscience » de son rapport à l'être, reste à savoir quel horizon conceptuel détermine couramment cette "conscience». La vue existentiale que constitue le "comprendre» qui, en tant qu'ouvrir, "concerne toujours la constitution fondamentale totale de l'être-au-monde" ", accueille l'être sur le mode de la préoccupation et donc s'oriente sur la dimension primitivement ustensiliaire du monde ontique au sens où le Dasein s'affaire, par conséquent s'ordonne au mode d'être de la Zuhandenheit. Cependant, c'est tout le mérite de l'analytique existentiale et de la phénoménologie heideggerienne que de mettre au jour le caractère originaire et archéo-ontologique de la Zuhandenheit. Rien n'est donc moins exprès que le mode d'être de la Zuhandenheit dont le sens d'être et les implications ontologiques pour le Dasein sont restés ignorés de la Tradition. Rien n'est donc plus « opaque » que la Zuhandenheit et pourtant, si la chose a toujours été présente sans jamais avoir été prise en vue, n'est-ce pas parce qu'elle est ce qu'il y a de plus «transparent»? Toutefois, le mode d'être qui a prévalu et qui s'est imposé comme ce qu'il y a de plus "évident» (Selbstverständlich) est la Vorhandenheit. C'est sur cette modalité que la Tradition philosophique s'est toujours orientée pour expliciter la détermination ontologique de l'étant en général et de l'être de l'homme. Mais la portée ontologique et la signification historiale de la Vorhandenheit, du fait même de son évidence, n'ont pas été dégagées. Comment, dès lors, la Vorhandenheit, étant donné son évidence, c'est-à-dire en un sens sa transparence, a pu dominer tout le discours ontologique?

Si cette modalité ontologique a pu s'imposer cela tient précisément au fait qu'elle n'a pas été appréhendée comme modalité et qu'elle est apparue comme le seul sens d'être patent dans la mesure où ce par quoi elle est saisie, à savoir la connaissance, résultant d'une déficience de la préoccupation ${ }^{4}$, n'ayant plus affaire qu'à l'aspect du monde n'accueille l'être qu'à l'aune de ce qui se donne dans la présence et comme simple subsistance ou substance. Si la Zuhandenheit est demeurée inaperçue c'est qu'elle n'a jamais été ontologiquement caractérisée. Or, cette absence de détermination ontologique le mode d'être le plus proche la doit à la non-problématisation de la Vorhandenheit comme modalité ontologique. Que la Zuhandenheit ait pu demeurer inquestionnée et non thématisée comme modalité ontologique cela tient à ses propres déterminations fondamentales. En effet, Heidegger montre que la modalité de donation de l'être de l'étant intramondain au sein de la mondanéité ambiante consiste en un retrait au sens où l'usage du Zuhanden, soit de l'étant-à-portée-de-la-main ou plus trivialement l'outil, est subordonné aux caractéristiques de retenue que sont la non-imposition (NichtAuffälligkeit), la non-saturation (Nicht-Aufsässigkeit) et la non-insistance (NichtAufdringlichkeit).

11 Ces déterminations négatives sont en réalité le signe du phénomène positif et originaire de la Zuhandenheit. La traduction concrète de ces déterminations négatives assure un usage optimal de l'outil qui se retire ainsi thématiquement au profit de son emploi : "moins la chose-marteau est simplement "regardée", plus elle est utilisée efficacement et plus originel est le rapport à elle, plus manifestement elle fait encontre comme ce qu'elle est-comme outil ${ }^{5}$ ». Dès lors, l'usage est ce qui est manifesté, c'est-àdire ce qui transparaît au détriment de cela même qui demeure transparent et qui 
laisse, pour cela, transparaître l'usage seulement, à savoir l'être-outil, le Zuhanden, et à plus forte raison l'être de l'étant intramondain, à savoir la Zuhandenheit.

Cependant, toute l'analyse de la mondanéité (qui déploie sa stratégie phénoménologique au fil des paragraphes $15,16,17,18)$ dont l'objectif est de mettre au jour l'antécédence ontologique de la Zuhandenheit montre d'une part qu'une telle mise au jour requiert la lecture phénoménologique d'une rupture du circuit ustensilier et d'autre part que la non-retenue de l'être-outil dans sa non-imposition implique corrélativement la survenue de la modalité ontologique de la Vorhandenheit et donc du caractère être-sous-la-main de l'étant-à-portée-de-la-main. C'est en ce sens que la Zuhandenheit est transparente. Elle laisse, en effet, transparaître l'usage, elle laisse passer la lumière de l'usage. Comme une vitre qui ne se voit pas mais qui laisse tout voir à travers elle, le caractère "transparent " de la Zuhandenheit ne se révèle qu'à l'occasion d'une rupture du réseau de renvoi ustensilier, partant n'apparaît qu'à l'occasion d'une brisure de la glace de l'être de l'étant intramondain tel qu'il se donne au sein du monde ambiant. Pour autant, déduire d'une telle rupture du contexte ustensilier l'annonce de la mondialité de l'être-à-portée-de-la-main, cela implique d'avoir saisi la dimension éminemment ontologico-positive de la préoccupation comme guise irrévocable et fondamentale de l'être-au-monde en sa quotidienneté. C'est la raison pour laquelle, cette dimension ayant été manquée, le thème phénoménal correct a toujours été recouvert par une qualification ontologique autre de l'être de l'étant intramondain qui s'est avérée régnante dans l'histoire de l'être et de la pensée : la Vorhandenheit.

13 Si cette dernière modalité ontologique s'est imposée comme LA détermination de l'être de l'étant c'est qu'il est toujours revenu à la connaissance, dont on sait qu'elle est une déficience de l'avoir-affaire préoccupé avec le monde, de régler primitivement et absolument le discours sur l'être. Partant, l'être de l'étant ne peut plus être reçu que sur le mode restant et négatif du connaitre qui n'accueille plus que le visage (eidos) de l'étant et n'a de compréhension que pour un sens d'être au sens de l'être-sous-la-main, de ce qui est présent là-devant. Lorsque l'étant dont il est question dans la préoccupation apparaît à l'occasion d'une défaillance, il est appréhendé sur le mode déficient de la connaissance et ne devient visible qu'en son être-sous-la-main : «Ce nonà-portée-de-la-main perturbe, et rend visible la saturation de l'objet premier et primaire de la préoccupation. Avec cette saturation s'annonce d'une façon nouvelle l'être-sous-la-main de l'étant-à-portée-de-la-main : c'est l'être de ce qui traîne encore et demande à être liquidé. Les modes de l'imposition, de l'insistance et de la saturation ont pour fonction de porter au paraître dans l'étant-à-portée-de-la-main le caractère de l'être-sous-la-main ${ }^{6}$.» Certes, par la suite Heidegger ne manque pas de spécifier que ce surgissement du caractère de l'être-sous-la-main demeure encore attaché à l'étant-àportée-de-la-main en son mode déficient ou manquant, mais ce qui importe pour nous, c'est de comprendre que la visibilité de l'étant est fondamentalement ordonnée à l'êtresous-la-main, soit à la Vorhandenheit. Dès lors que le connaître comme réflexion noncirconspecte et comportement théorique entre en jeu, le pur et simple avisement (Hinsehen) qu'il implique ne lui donne à voir et, ce qui revient au même, à penser que du sous-la-main en son être-sous-la-main. Sitôt que l'identification entre voir et penser est établie sans être le moins du monde interrogée en sa légitimité ni même problématisée, l'étant apparaît dans la guise de l'être-sous-la-main. 
14 Aussi, si la Zuhandenheit est, comme nous l'avons vu, ce qui est transparent et si, comme nous le pensons, l'opacité de cette modalité ontologique vient du fait qu'elle est précisément transparente et laisse donc voir à travers elle ce qui se donne à voir, en revanche, ce qui est pris en vue au travers du transparent dès lors que la réflexion entre en jeu dans la modalité du connaître, c'est-à-dire dès lors qu'une pensée s'élabore au sujet de l'être, n'est rien d'autre que la transparence du transparent, dont on sait à présent qu'elle relève de la Vorhandenheit. En effet, ce qui donne à penser, ce qui s'offre à la pensée, ou plus exactement, à la pensée dans la modalité dérivée du connaître est la transparence du transparent. C'est cela qui est dès l'abord thématique et thématisable pour le connaître. C'est pourquoi, fondamentalement, la Zuhandenheit n'a pas été thématisée et appréciée comme modalité ontologique, pourquoi encore, en dernière instance, la Vorhandenheit n'a pas été appréhendée comme modalité ontologique puisque saisie comme seul sens d'être pertinent. Tout se passe comme si la réflexion, la ratio de l'homme, était obnubilée par la Vorhandenheit, cette dernière "faisant écran" empêchant ainsi de saisir thématiquement le sens d'être immémorial de la Zuhandenheit tout en protégeant en même temps la non-imposition de l'étant-à-portée-de-la-main tel qu'il fait encontre dans le monde ambiant. Cet écran est d'autant plus efficace qu'il ne se présente pas comme écran mais s'offre à la réflexion et à l'intelligence comme transparence, comme ce qui, par conséquent, rend possible a priori toute visibilité.

Telle est, peut être, l'une des pistes explicatives à l'une des ultimes questions que se pose Heidegger alors qu'il interrompt Être et temps : «pourquoi l'être est-il justement "de prime abord" "conçu" à partir du sous-là-main et non pas à partir de l'à-portée-dela-main, qui pourtant se trouve encore davantage à proximité ? ». Or, si la Vorhandenheit assure constamment sa souveraineté cela tient au fait qu'elle règne sur le visible et que toute l'histoire de la pensée est commandée par le sens de la vue. Heidegger, loin d'ignorer ce phénomène le relève et assume cette donne historiale en reprenant délibérément la notion de "vue » telle qu'elle transcende le « comprendre " (Verstehen) en tant que translucidité «Durchsichtigkeit»: "Afin de maintenir la connexion avec elle (la tradition), on peut formaliser les concepts de vue et de voir de manière à les prendre comme des termes universels caractérisant tout accès - en tant qu'accès - à l'étant et à l'être ${ }^{8}$ ».

sous couvert de "formalisation ", n'était-il pas inévitable, réintroduisant la thématique de la vue en son origine existentiale, de voir se maintenir subrepticement et clandestinement la domination silencieuse de la Vorhandenheit? Puisque, selon Heidegger, seule compte "cette propriété spécifique du voir", à savoir le fait qu'il " laisse faire encontre en lui-même à découvert l'étant qui lui est accessible ${ }^{9}$ » et que cela chaque "sens" le fait "évidemment à l'intérieur de son domaine natif de découverte ${ }^{10}$ » n'eut-il pas été phénoménologiquement opportun et fructueux d'élire un autre "sens" afin de formaliser l'accès à l'étant et à l'être ou, même encore, de problématiser ontologiquement le sens des «sens »? Peut-on légitimement parler de propriété spécifique là où d'autres "sens" partagent, dans leurs domaines, une telle propriété ? La maintenance, par Heidegger, de la vue et du voir comme mode d'accès à l'étant et à l'être, par delà le motif invoqué de resituer la vue sensible ou encore noétique en leur origine existentiale, trouve sa légitimité dans la détermination archaïque (c'est-à-dire, grecque) du logos comme « faire-voir ».

17 En effet, originellement, logos signifie tout autant que "déloun", il donne à voir, possède une fonction monstrative. Le logos est apophantique, " phaïnestique », telle est 
la fonction primaire du logos. Autrement dit, la phénoméno-logie, science de l'être de l'étant, méthode privilégiée pour la conquête du sens de l'être, est toute entière traversée par l'archi-thème du voir. Dans la mesure où elle se déploie dans l'orbe du visible, pèse donc sur elle, en sa signification fondamentale, la menace d'une assignation radicale à la Vorhandenheit. Partant, cette emprise, voire cette hantise de la Vorhandenheit ne peut que troubler le discours phénoménologique dans sa tentative d'élucidation du sens de l'être.

Ce brouillage du discours phénoménologique est singulièrement perceptible à l'endroit du «passage » du sens d'être au sens de l'être-à-portée-de-la-main (Zuhandenheit) au sens d'être de l'être-sous-la-main (Vorhandenheit). En effet, comme nous l'avons vu, l'apparition phénoménologique de la Zuhandenheit ne va pas sans quelque surgissement de la Vorhandenheit. Or, sitôt que la Vorhandenheit règne, elle voile le caractère ontologique de la Zuhandenheit. Le basculement ontologique de la Zuhandenheit à la Vorhandenheit, que Heidegger pense dans les termes du « virage » (Umschlag), s'effectue en un clin d'œil, dans la magie d'un instant. Sans doute est-ce là le point d'orgue de l'opacité ontologique de la transparence dans la mesure où cette modification ontologique fondamentale, se déployant pourtant sur la scène privilégiée du logos, se passe en silence et invisiblement, là où tout devrait s'entendre et se voir. Resserrons l'angle pour mieux saisir ce phénomène de l'Umschlag.

Ce terme de virage, «Umschlag ", apparaît de façon exemplaire à deux reprises dans Être et Temps, notamment au $\S 33$ et $\S 69 \mathrm{~b}$, lorsqu'il s'agit d'expliciter rien de moins que le passage d'une compréhension d'être (la Zuhandenheit, qualifiée d'originaire) à une autre (la Vorhandenheit, qualifiée de dérivée). La compréhension de l'articulation de ces deux modalités ontologiques n'en devient que plus impérieuse. L'Umschlag non seulement signale le passage de la préoccupation circonspecte à la découverte théorique du "sous-la-main », mais indique aussi le passage d'un parler circonspect (ordonné à la Zuhandenheit) à un parler modifié (orienté à la Vorhandenheit). Or il s'agit d'une étrange «modification» du parler puisque ce qui est modifié ce n'est pas tant le parler que «le fait que nous considérons "à neuf" l'à-portée-de-la-main comme sous-la-main ${ }^{11}$ ». Pourtant Heidegger met l'accent sur une modification du parler, puisqu'il est question, par la suite, d'un énoncé qualifié de " physique ». La césure est donc, en droit, réalisable entre un " parler » circonspect et un parler «théorique » et ce pour une même phrase, par exemple : « le marteau est lourd ». En passant d'un parler à un autre, on passe d'un monde à un autre, et cependant la différence est inaudible, invisible, indécelable à la lecture et à l'écoute. Soit la transparence même.

Le virage, selon Heidegger, ne s'effectue pas, comme une lecture rapide du § 33 pourrait le laisser penser, d'un maniement muet à une situation énonciative, mais d'une expression ordonnée à la circonspection à une proposition énonciative telle qu'elle est comprise par la logique classique. Ne pourrait-on pas mettre en question, non pas la légitimité de cette duplicité régnante au cœur de l'énoncé, mais la possibilité d'une reconnaissance assurée de telle ou telle situation? Sur quelle détermination ontique le départ entre parler circonspect et parler modifié peut-il se faire ? Cette question ne se limite pas au plan ontique puisqu'elle questionne aussi la rigoureuse "pureté » d'une distinction ontologique entre Zuhandenheit et Vorhandenheit. L'Umschlag, le "virage " s'effectue au fil du logos, plus précisément au fil d'une « considération » exclusive d'un certain logos, et opère le passage d'une compréhension d'être à une autre. Heidegger suppose qu'avant cette « nouvelle » considération de l'a-portée-de-la-main, avant que tel 
étant (le marteau) devienne « objet » d'un « énoncé », la Zuhandenheit était " pure » de toute Vorhandenheit.

Si l'on peut légitimement mettre en question la rigoureuse distinction entre le parler circonspect et le parler modifié, par conséquent, cela ne nous mène-t-il pas à douter de la pureté supposée de la Zuhandenheit, et partant à douter de son antécédence ontologique et de son caractère immémorial? Notre propos, ici, n'était pas d'engager une analyse critique de la phénoménologie heideggerienne afin de saisir les présupposés de cette dernière, mais il s'agissait seulement de montrer le caractère éminemment énigmatique et obscur du concept ou plutôt de la notion de transparence dont nous avons montré la plurivocité des acceptions en même temps que l'unité ontologique et existentiale de sa signification. De même qu'un prisme donne lieu à un jeu bigarré de lumière et de couleurs, l'être, prisme obscur, en son retrait et en sa retenue donne lieu à l'étrange miroitement des transparences de l'être de l'étant.

Si la "transparence appropriée", nécessitant la destruction de l'histoire de la métaphysique, ne peut être conquise, cela tient à des raisons fondamentales qui relèvent de la constitution de l'être même et auxquelles le Dasein ne peut prendre part qu'au titre d'étant questionnant pour qui, singulièrement dans le tout de l'étant, il y va de son être. Dans le « il y a » de la question de l'être, dans la possibilité même d'ouvrir un questionnement eu égard à l'être, la transparence, qui voile toute chose en mimant la clarté absolue, mine dès l'abord la possibilité d'une réponse quant au sens de l'être en contaminant le questionnant en son être même. Ainsi, point pour nous la duplicité de l'être de l'étant à travers l'opacité ontologique de ses transparences.

\section{NOTES}

1. Heidegger Martin, Être et temps § 2, Emmanuel Martineau (trad.), Paris, Authentica, 1985, 355 p.

2. Heidegger Martin, Être et temps $\S 31$, Emmanuel Martineau (trad.), Paris, Authentica, 1985, $355 \mathrm{p}$.

3. Heidegger Martin, Etre et temps $§ 31$, Emmanuel Martineau (trad.), Paris, Authentica, 1985, $355 \mathrm{p}$.

4. Heidegger Martin, Être et temps § 13, Emmanuel Martineau (trad.), Paris, Authentica, 1985, 355 p. «Pour que devienne possible le connaître en tant que détermination considérative du sous-la-main, il est préalablement besoin d'une déficience de l'avoir-affaire préoccupé avec le monde ".

5. Heidegger Martin, Être et temps § 15, Emmanuel Martineau (trad.), Paris, Authentica, 1985, $355 \mathrm{p}$.

6. Heidegger Martin, Etre et temps § 16, Emmanuel Martineau (trad.), Paris, Authentica, 1985, $355 \mathrm{p}$.

7. Heidegger Martin, Être et temps § 83, Emmanuel Martineau (trad.), Paris, Authentica, 1985, $355 \mathrm{p}$.

8. Heidegger Martin, Etre et temps § 31, Emmanuel Martineau (trad.), Paris, Authentica, 1985, $355 \mathrm{p}$. 
9. Heidegger Martin, Être et temps §31, Emmanuel Martineau (trad.), Paris, Authentica, 1985, $355 \mathrm{p}$.

10. Heidegger Martin, Etre et temps $§ 31$, Emmanuel Martineau (trad.), Paris, Authentica, 1985, $355 \mathrm{p}$.

11. Heidegger Martin, Être et temps § 69b, Emmanuel Martineau (trad.), Paris, Authentica, 1985, $355 \mathrm{p}$.

\section{RÉSUMÉS}

Y a-t-il une dimension ontologique de la transparence? Avant de recevoir, dans le cadre de l'ontologie phénoménologique de Sein und Zeit, une fonction conceptuelle nette au sein de la terminologie de l'analytique existentiale (§31), la transparence (Durchsichtigkeit) a fait son apparition lors de l'explicitation de la question du sens de l'être (\$2). Question fondamentale par excellence, la question du sens de l'être exige, pour être posée, la considération de son histoire propre, histoire dont Heidegger nous dit qu'elle requiert une «transparence appropriée » (angemessenen Durchsichtigkeit). Cette "transparence appropriée " n'est rien d'autre que la tâche même de la destruction de l'histoire de l'ontologie. Or une telle tâche, on le sait, n'a pu être menée à bon terme puisqu'elle correspond au programme de la deuxième partie de Sein und Zeit et que seules les deux premières sections de la première partie de l'ouvrage ont été publiées. Si la transparence est une tâche c'est que règne premièrement une certaine opacité. Constater que la transparence, en l'espèce d'une destruction de l'histoire de l'ontologie, n'a pu être conquise, cela ne doit-il pas signifier qu'il y a lieu de prendre acte d'une opacité ontologique de la transparence? Traditionnellement marquée du sceau de «l'évidence » (Selbstverständlichkeit) la question du sens de l'être est tombée dans l'oubli. En ce sens, la transparence ou l'évidence de l'être dissimule l'opacité ou l'obscurité de son sens propre. Nous pouvons dire, dès lors, que la transparence ontique de l'être, entendons l'évidente compréhension que nous en avons dans l'usage quotidien, se double et se paye d'une obscurité ou opacité de son sens ontologique. Or il s'agira, pour nous, de montrer que l'évidence de l'être ainsi que l'obscurité de son sens ontologique trouve son origine dans la transparence du sens d'être qui sans cesse s'impose comme premier, à savoir celui-là même contre lequel tous les efforts phénoménologiques de Sein und Zeit sont tendus : la Vorhandenheit. Nous verrons en quoi le sens d'être plus immémorial de la Zuhandenheit est lui-même hanté par la Vorhandenheit, partant par l'opacité ontologique de la transparence. Il conviendra d'élucider le lien essentiel qui relie cette prime apparition de la transparence (§ 2) à celle du paragraphe (\$31) consacré au comprendre du Dasein dans lequel cette dernière est promue au rang de détermination existentiale. Pourquoi, en effet, un même terme vient qualifier la vue propre du Dasein en tant qu'il se comprend authentiquement et la tâche de la destruction de l'histoire de l'être? Nous verrons en quoi rendre sa transparence à l'histoire de l'ontologie implique l'exhibition de l'étrange opacité ontologique de la transparence ou des transparences de l'être. Comprendre pourquoi l'analytique existentiale est toute entière transie par la question de la transparence et comment un tel thème travaille non seulement toute l'histoire de la métaphysique via la Vorhandenheit, mais aussi l'ontologie phénoménologique à travers le jeu du passage (Umschlag) articulant les deux sens d'être de la Zuhandenheit et de la Vorhandenheit, tel sera l'enjeu de notre propos. 
INDEX

Personnes citées: Heidegger (Martin)

Mots-clés : Dasein, existence, hantise, histoire de l'être, ontologie, opacité, phénoménologie, transparence

\section{AUTEUR}

\section{HERVÉ BONNET}

Chercheur en histoire de la métaphysique occidentale et phénoménologie, il prépare les concours d'agrégation et du CAPES de philosophie. 